\title{
Ice crystals grown from vapor onto an orientated substrate: application to snow depth-hoar development and gas inclusions in lake ice
}

\author{
Edward E. ADAMS, Daniel A. MILLER \\ Department of Civil Engineering, Montana State University, Bozeman, Montana 59717-3900, U.S.A. \\ E-mail:eda@ce.montana.edu
}

\begin{abstract}
A laboratory experiment was conducted in which new ice crystals were nucleated from the vapor phase onto large existing ice crystals obtained from Antarctic lake ice. Flat, smooth ice-crystal surfaces were prepared, with $c$ axes oriented either vertically or horizontally. When these were subjected to a supersaturated vapor environment, multiple individual crystals nucleated onto the substrates adopting the same crystallographic orientation as the parent. A dominant grain-growth scenario for kinetic-growth metamorphism in snow, which in some ways is analogous to the oriented morphologies in lake ice, is hypothesized. In the lake-ice-growth scenario, optimally oriented crystals will grow at the expense of those less preferentially positioned. The proposed dominant grain-grow th theory for snow is in agreement with the observed decrease in the number of grains and the proximal similarity of crystal habit in kinetic-growth metamorphism in snow. Similarly, kinetic crystal growth on the interior of gas inclusions in Antarctic lake ice will also acquire the crystallographic orientation of the substrate ice. These small-faceted interior crystals significantly influence light scattering and penetration in the lake-ice cover.
\end{abstract}

\section{INTRODUCTION}

Ice has a hexagonal crystallographic structure, which is defined by three $a$ axes orientated at $120^{\circ}$ to one another on the "basal plane" with the $c$ axis defined as normal to this plane. Perpendicular to the basal plane (parallel to the $c$ axis) are the six "prism faces".

Water freezing from the liquid state onto an existing ice crystal will tend to take on the crystallographic orientation of the substrate. In the formation and growth of ice covers, several different crystallographic structures may develop (Michel and Ramseier, 1971). For example, ice covers grown slowly under quiescent conditions on lakes have a propensity toward a $c$-axis vertical orientation (Gow and Langston, 1977; Lock, 1990; Petrenko and Whitworth, 1999). The nomenclature on lake ice describes this $c$-axis vertical structure as $\mathrm{S} 1$ ice. The $\mathrm{S}$ represents a secondary growth pattern, which follows the primary, P, formation (Michel and Ramseier, 1971). However, an ice cover formed and grown more rapidly from the liquid phase has a preferentially higher growth rate parallel to the basal plane (Gow and Langston, 1977; Lock, 1990; Petrenko and Whitworth, 1999). Therefore, a vertically oriented basal plane will grow deeper into the liquid phase than an adjacent $c$-axis-vertical crystal. As this process progresses, the temperature gradient and thus the heat-conduction rate in the ice at the tip of the vertically oriented basal plane is increased, further benefiting its natural growth advantage. Basal-plane-growth dominance results in the crowding out of $c$-axis-vertical crystals, and the ice sheet may develop with a $c$-axis-horizontal preference, referred to as S2 ice. The crystals which comprise the secondary ice are larger and fewer in number than those of the primary ice (Petrenko and Whitworth, 1999).

Snow on the ground will undergo significant morphological changes following its initial deposition. The crystal forms resulting from two different thermal environments generally categorize metamorphism in a "dry" snowpack, where vapor-diffusion processes are generally considered to dominate. In the absence of a "significant" temperature gradient, rounded well-sintered grains develop, resulting in a snowpack with a generally high structural integrity. This is commonly referred to as the equilibrium-growth form. In the presence of a sufficient temperature gradient, nominally taken to be $10-25^{\circ} \mathrm{Cm}^{-1}$ (Akitaya, 1974; Armstrong, 1980; Marbouty, 1980; Colbeck, 1983a,b), snow metamorphoses into a structure composed of large faceted crystals, known as depth hoar or the kinetic-growth form. This process is referred to as kinetic-growth, temperature-gradient metamorphism, or recrystallization. This often results in a snowpack of low structural integrity yielding increased avalanchehazard and vehicle-mobility problems. It has been suggested that in this metamorphic process, the total number of crystals decreases (Colbeck, 1983b; Sturm and Benson, 1997). Given sufficient time, chains of cup-shaped crystals (usually partial cups) may form with the open end downward, accompanied by complimentary pore channels parallel to the vertical temperature gradient (Trabant and Benson, 1972; Akitaya, 1974, Adams and others, 1995).

The importance of crystal growth from vapor in existing ice structures is not limited to snow. Studies in the McMurdo Dry Valley lakes of Antarctica indicate that in the presence of temperature gradients, the interior top of gas bubbles in the 


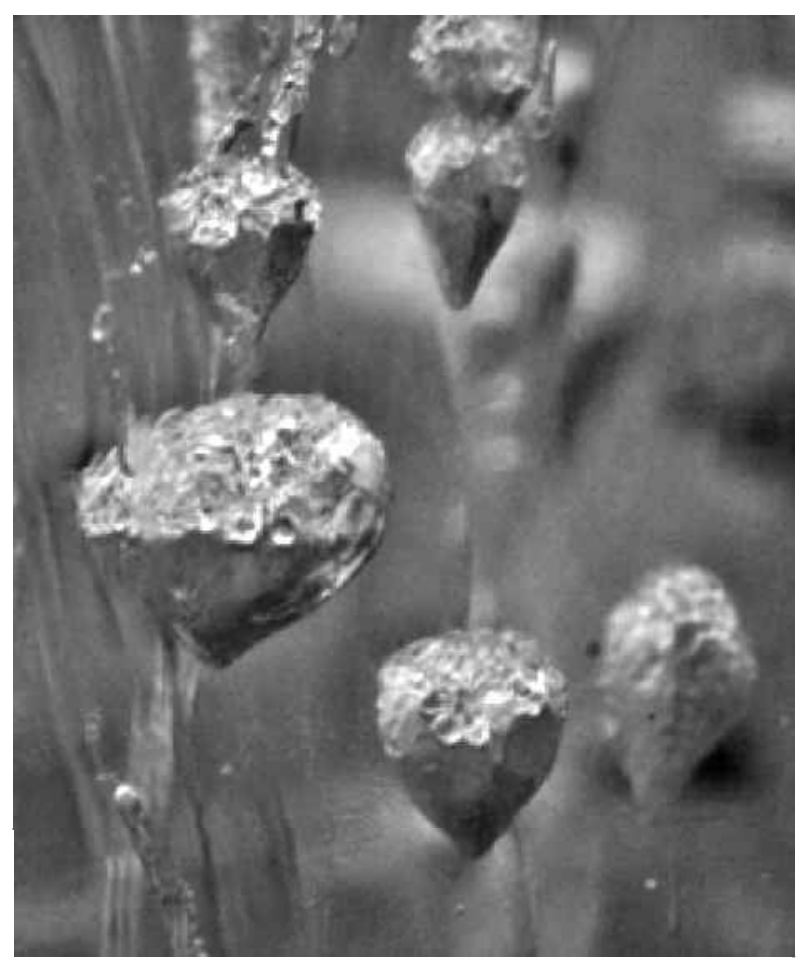

Fig. 1. Typical inverted teardrop and fine tube-like bubbles that predominate in the upper regions of the McMurdo Dry Valley lake-ice covers. This sample is from Lake Bonney, a liquidbased lake. The largest bubble is approximately $2 \mathrm{~mm}$. The crystal growth displayed here, on the top sections of the bubbles, is common. Temperature gradients drive this crystal growth within the bubbles.

ice develop frost (Fig. 1) (Adams and others, 1998). This causes light scattering, thus affecting the amount of photosynthetically active radiation and thermal energy that is available for microbial communities in the ice and lake water below (Priscu and others, 1998).

In the case of atmospheric ice crystals formed from vapor, it is the temperature that primarily determines the habit. Secondary growth features (such as dendrites developing along the $a$ axis on plate crystals) are determined by the degree of vapor supersaturation ( Nakaya and others, 1938; Hanajima, 1949; Kobayashi, 1957; Lamb and Hobbs, 1971; Hobbs, 1974). Crystals grown from the vapor phase have been examined using various fibers (Nakaya and others, 1938; Hallet and Mason, 1958), and other nucleation sources (Aufm Kampe and others, 1951; Fukuta, 1968; Schaefer and Cheng, 1968), including glass (Kobayashi, 1957) and metal substrates (Isono and Iwai, 1969; Lamb and Hobbs, 1971). Surprisingly, to our knowledge, the habit of crystals grown from vapor onto ice substrates has not been explicitly demonstrated in the literature. A laboratory study was designed to verify the supposition that heterogeneous ice nucleation and growth from the vapor phase will result in crystals with the same crystallographic orientation as a large crystal substrate. Verification that accreted crystals do inherit parentcrystal orientation will lead to an enhanced understanding of depth-hoar development, as well as mass-adhesion processes on cavities in the interior of ice sheets.

\section{METHODOLOGY}

The McMurdo Dry Valley lakes are a unique ecosystem of

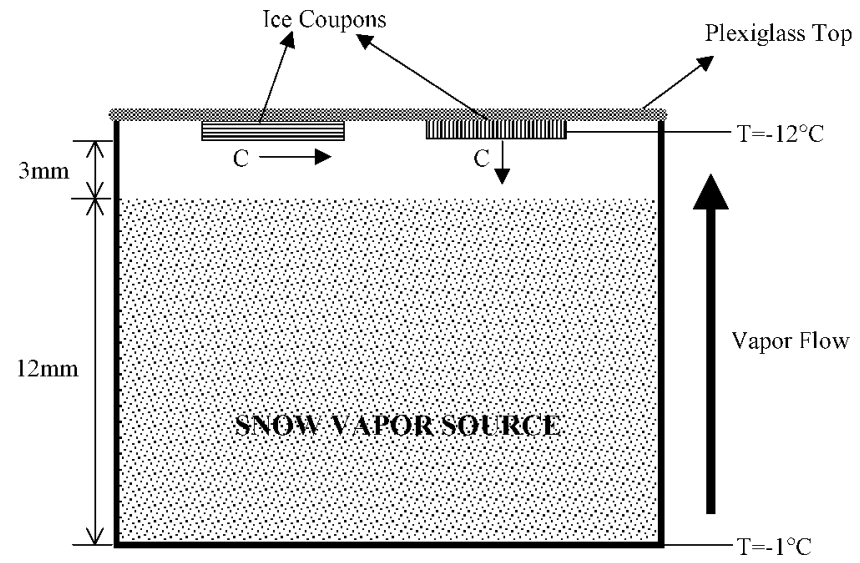

Fig. 2. Crystal-growth experiment configuration and conditions.

perennially ice-covered liquid-based lakes (Priscu and others, 1998). One such lake, Lake Bonney, has about $4 \mathrm{~m}$ of ice cover composed (at least in the lower regions) of S1 ice (Adams and others, 1996). Since it is composed of large oriented crystals, an ice-core sample from Lake Bonney was used as substrate for the crystal-growth experiment. A universal stage for ice (Langway, 1958) was employed to determine the crystallographic orientation and to verify the S1 structure of a sample from $2.4 \mathrm{~m}$ below the lake-ice surface. From the $\mathrm{S} 1$ ice, two thin (approximately $2 \mathrm{~mm}$ thick) plates were cut as coupons from the $8 \mathrm{~cm}$ diameter core. One of the ice coupons was cut so the $c$ axis was contained in the thin plane of the sample while the other coupon had the $c$ axis perpendicular to the thin dimension (accuracy estimated at $\pm 5^{\circ}$ ). The ice samples were then mounted on a Plexiglass plate. Once attached to the Plexiglass, the samples were melted slightly by simultaneously placing the exposed faces of both ice samples in contact with a "warm" aluminum plate. This provided a smooth face as well as a uniform thickness for both ice coupons. The thin liquid layer resulting from this melt adopted the crystal orientation of the substrate upon freezing, which was verified using cross-polarization. The experiment was also executed when the ice surface was exposed using a razor blade and no melting.

A small, insulated box was constructed in which the Plexiglass plate served as a top seal (Fig. 2). The insulation served to minimize horizontal temperature gradients. Snow was placed in the box with a $\sim 3 \mathrm{~cm}$ gap between the snow and the ice samples. The package was placed on a $-1{ }^{\circ} \mathrm{C}$ plate in a $-17^{\circ} \mathrm{C}$ cold laboratory to establish a temperature gradient and thus a supersaturated environment at the ice coupon. Although vapor pressure was not actually measured, the resulting kinetic crystal growth implied supersaturation relative to the ice surface. One ice sample's $c$ axis was oriented parallel to the temperature gradient (vertical) and the other's perpendicular to the temperature gradient (horizontal). The ice temperature, measured by placing a type $\mathrm{T}$ thermocouple in contact with the ice, remained near $-12{ }^{\circ} \mathrm{C}$ throughout the experiment, even during the periodic defrost cycles of the cold room.

Crystal development under the conditions described was examined daily by removing the Plexiglass plate with the attached ice coupons from the insulated box. Crystals were examined with a binocular optical microscope and photographed periodically. The experiment was repeated several times with each test lasting 7-10 days. 

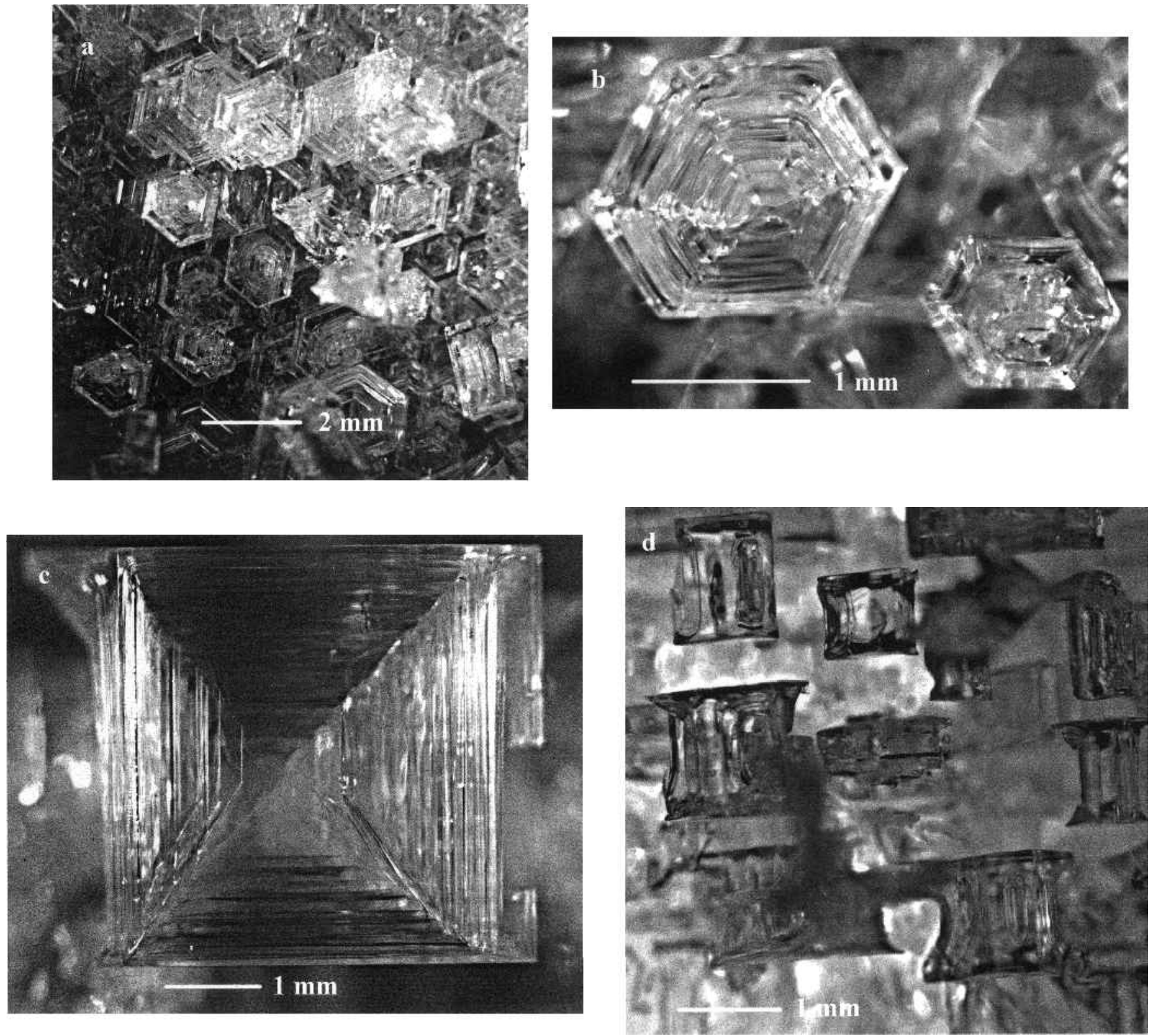

Fig. 3. Ice crystals grown from vapor phase onto existing ice substrates. View is normal to substrate plane. ( $a, b)$ formed on crystals with $c$ axis parallel to the temperature gradient. $(c, d)$ formed on crystals with the c axis perpendicular to the temperature gradient.

\section{RESULTS}

Examination of the substrate coupons after a few days revealed multiple individual crystals on each of the large, flat $c$-axis- vertical and -horizontal crystal surfaces. These crystals developed with the same orientation as the large substrate parent crystals in both $c$-axis configurations (horizontal and vertical) (Fig. 3). In the $c$-axis-horizontal sample (Fig. 3c and d), the crystal $c$ axes are also parallel, which is analogous to $\mathrm{S} 3$ ice grown from the liquid. S3 ice is defined as the $c$ axes being parallel as well as horizontal (Petrenko and Whitworth, 1999). The hexagonal orientations of the $c$-axis-vertical crystals (Fig. $3 \mathrm{a}$ and b) are also uniformly oriented with regard to the six prism faces. The hopper-type crystals, which are manifest as hollow prismatic columns and dish-shaped hexagonal plates (Mason and others, 1963), were well developed both on the basal and prism faces (Fig. 3b and d). It should be noted that scratches, corners and imperfections all showed enhanced growth rates. In fact, the well-developed prism-face crystal seen in Figure $3 \mathrm{c}$ developed on an edge of the $c$-axis-horizontal ice coupon. Even across the flat, relatively uniform, substrate surfaces there was variation in growth rates. Off the ice coupons, on the Plexiglass substrate, crystals also developed, but no preferential orientation could be discerned. Well-developed cup shapes were not apparent on the Plexiglass, although partial cups and columns were evident.

Figure 4 is an in-situ image of several faceted crystals produced on the ice coupons. Notice that the crystals are not touching, but complete development is impeded, presumably by the competition for a limited vapor supply.

\section{DISCUSSION}

In light of this study, we hypothesize about morphological processes in a snowpack. We conjecture that, in general, atmospherically formed snow crystals will be deposited onto the ground with a random crystallographic orientation. This is analogous to randomly oriented P4 (primary type 4) crystal development in lake ice, which develops from nucleation of snow that falls on the liquid-water surface (Petrenko and 


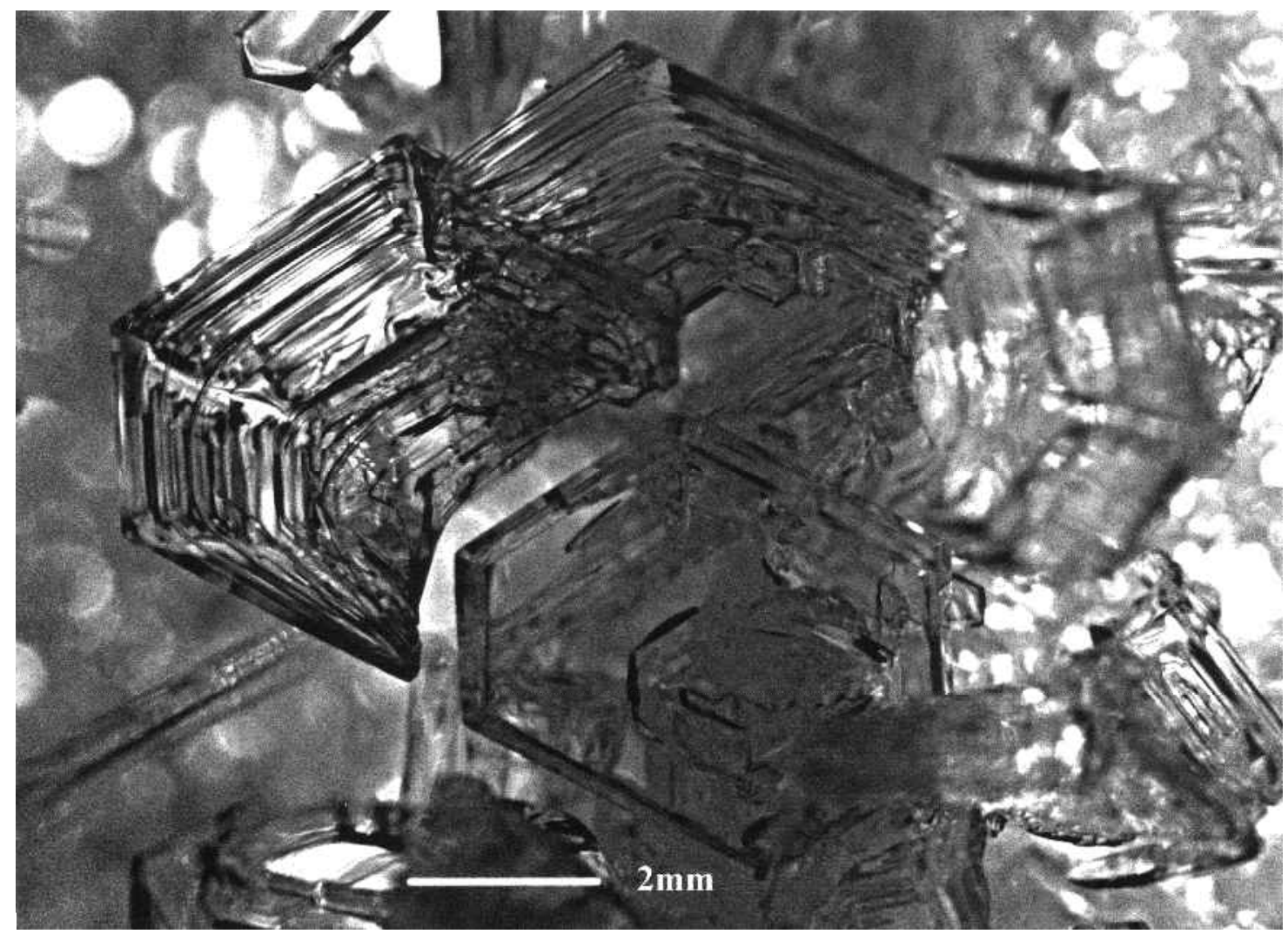

Fig. 4. Crystals competing for limited vapor resulting in incomplete growth.

Whitworth, 1999). Further to this, we speculate about secondary morphologies that develop in a snowpack. When subjected to a temperature gradient, the "new" crystals acquire the orientation of the substrate snow grains onto which they accrete. A particular kinetic-growth crystal is at an advantage if the substrate crystal orientation is better situated to grow by extending downward into warmer pore vapor unimpeded by neighboring grains. As in the development at the S2 freezing front in the liquid environment, this will establish a larger temperature gradient and faster growth rate at the tip of the crystal. Developing crystals that are favorably oriented would crowd out those with less advantage. In a natural snowpack, fully developed cups may be relatively rare due to the close proximity of other grains. Identification of crystallographic orientation is not as obvious in the case of partial cups.

In the presence of a sufficiently large temperature gradient, and thus mass flux, we hypothesize that numerous heterogeneous nucleations will activate on each grain, inheriting that orientation, as was demonstrated in this study on large crystallographically oriented substrates (Fig. 3). When a locally preferred crystal-growth environment dominates, less-ideally oriented crystals will have their advance curtailed.

Depth-hoar crystal morphologies within the same region of a snowpack are of similar habit (Akitaya, 1974) and are correlated with temperature and temperature gradient. This, coupled with the fact that crystals grow with the same orientation as the substrate grain, makes a dominantgrain theory (analogous to lake-ice development) plausible. The reported reduction in the number of grains (Colbeck, 1983b; Sturm and Benson, 1997) during kinetic growth in a snow pack is supported by the dominant-grain theory.

Depth-hoar crystals are similar in morphology to hopper crystals that develop in the atmosphere due to high supersat- uration and which grow on facial edges and corners where temperature and concentration gradients are greatest (Lock, 1990). The hollow or partial cups commonly described in a snowpack (Akitaya, 1974) imply a significant supersaturation leading to these secondary features. They can develop on either the basal or the prism faces (Fig. 3). However, the actual orientation may not be readily apparent in a snowpack, since surrounding crystals usually impede their full development (Fig. 4).

In addition, the experimental result has implications where ice accretes from the vapor phase onto an existing ice surface (e.g. bubbles in lake ice). Light-scattering bubbles on top of the interior of the air bubbles in lake ice will also adopt the orientation of the parent crystal at the top of the bubble when subjected to a temperature gradient (Adams and others, 1998). The development of the highly light-scattering steps depends on the supersaturation.

\section{CONGLUSION}

It has been clearly verified that an ice substrate, subjected to a supersaturated water-vapor environment, will nucleate numerous crystals across its surface with the same crystallographic orientation as the parent substrate.

With regard to the morphology of a snow cover subjected to a temperature gradient, we suggest that there is potential for a preferred structure to develop, analogous to that often seen in lake and sea ice, where the ice cover has a distinct crystallographic orientation. A fresh deposit of snow, or snow metamorphosed to the equilibrium form, will be composed of randomly oriented crystal grains. However, when such a configuration is subjected to a large temperature gradient, the ensuing supersaturation will induce the nucleation of 
numerous crystals onto the existing ice-grain network. Depending on the temperature and level of supersaturation, a particular crystal habit grown from the vapor will be preferential. Some of these developing crystals will naturally be sited within a region of the snowpack more optimally due to the orientation of the substrate or parent crystal. This, when coupled with an ice-grain/pore-space configuration that affords space in the preferred growth direction, results in some of the crystals being at a developmental advantage. As the optimally sited growing crystals progress, others will have their development arrested in the competition with the preferred-configuration crystals. This reduces the number of grains and leads to a (locally) crystallographically oriented snow cover.

This has implications for cold-region limnology since the development of highly faceted kinetic-growth crystals on the interior of vapor inclusions in lake ice are similarly prescribed. Configurations of the faceted crystals in the bubbles that develop in the presence of a temperature gradient impact the optical properties of the ice cover as a whole.

An enhanced perception of small-scale mass transfer and recrystallization processes evident in snow and ice inclusions are the thrust of this study. We feel that the microstructural morphology and the processes that govern its metamorphism are of paramount importance in understanding the properties of snow and ice and the macroscopic consequences. While the hypothesis presented seems to be supported by observations made to date, additional observational and quantitative work at the microstructural level is warranted in order to verify and elaborate these conjectures.

\section{ACKNOWLEDGEMENTS}

The research reported here was supported by the Geosciences Program of the U.S. Army Research Office under grant DAAG55-98-1-0018 and U.S. National Science Foundation grant OPP-9815512.

\section{REFERENGES}

Adams, E. E., J. C. Priscu and A. Sato. 1995. Some metamorphic processes in the lake ice of the McMurdo Dry Valleys. Antarct. F. U.S., 30(5), Review 1995, 307-309.

Adams, E. E., D. C. Vandervoort, M. Q. Edens and R. M. Lang. 1996. Ice grain orientation in processed snow. In Agrawal, K. C., ed. Proceedings of the International Symposium on Snow and Related Manifestations, SNOWSYMP 94, Manali, 26-28 September 1994. Manali, India, Snow and Avalanche Study Establishment, 96-102.
Adams, E. E., J. C. Priscu, C. H. Fritsen, S. R. Smith and S. L. Brackman. 1998. Permanent ice covers of the McMurdo Dry Valley lakes, Antarctica: bubble formation and metamorphism. In Priscu, J. C., ed. Ecosystem dynamics in a polar desert: the McMurdo Dry Valleys, Antarctica. Washington, DC, American Geophysical Union, 281-295. (Antarctic Research Series 72.)

Akitaya, E. 1974. Studies on depth hoar. Contrib. Inst. Low Temp. Sci., Ser. A 26.

Armstrong, R. L. 1980. An analysis of compressive strain in adjacent temperature-gradient and equi-temperature layers in a natural snow cover. f. Glaciol., 26(94), 283-289.

Aufm Kampe, H. J., H. K. Weickmann and J. J. Kelly. 1951. The influence of temperature on the shape of ice crystals growing at water saturation. $\mathcal{f}$. Meteorol., 8(3), 168-174.

Colbeck, S. C. 1983a. Ice crystal morphology and growth rates at low supersaturations and high temperatures. F. Appl. Phys., 54(5), 2677-2682.

Colbeck, S. C. 1983b. Theory of metamorphism of dry snow. 7. Geophys. Res., 88(C9), 5475-5482.

Fukuta, N. 1968. Some remarks on ice nucleation by metaldehyde. In International Conference on Cloud Physics, August 26-30, 1968, Toronto, Ont., Canada. Proceedings, 194-198.

Gow, A. J. and D. Langston. 1977. Growth history of lake ice in relation to its stratigraphic, crystalline and mechanical structure. CRREL Rep. 77-1.

Hallett, J. and B. J. Mason. 1958. The influence of temperature and supersaturation on the habit of ice crystals grown from the vapour. Proc. R. Soc. London, Ser. A, 247(1251), 440-453.

Hanajima, M. 1949. On the growth conditions of man-made snow. Low Temp. Sci., Ser. A 2, 23-29.

Hobbs, P.V. 1974. Ice physics. Oxford, Clarendon Press

Isono, K. and K. Iwai. 1969. Growth mode of ice crystals in air at low pressure. Nature, 223, 1149-1150.

Kobayashi,T. 1957. Experimental researches on the snow crystal habit and growth by means of a diffusion cloud chamber. F. Meteorol. Soc. Jpn, 75th Anniversary, 38-47.

Lamb, D. and P.V. Hobbs. 1971. Growth rates and habits of ice crystals grown from the vapor phase. F. Atmos. Sci., 28(8), 1506-1509.

Langway, C. C., Jr. 1958. Ice fabrics and the universal stage. SIPRE Tech. Rep. 62.

Lock, G. S. H. 1990. The growth and decay of ice. Cambridge, etc., Cambridge University Press.

Marbouty, D. 1980. An experimental study of temperature-gradient metamorphism. 7. Glaciol., 26(94), 303-312.

Mason, B. J., G.W. Bryant and A. P. van den Heuval. 1963. The growth habits and surface structure of ice crystals. Philos. Mag., 8, 505-526.

Michel, B. and R. O. Ramseier. 1971. Classification of river and lake ice. Can. Geotech. 7., 8(1), 36-45.

Nakaya, U., I. Sato and Y. Sekido. 1938. Preliminary experiments on the artificial production of snow crystals. 7. Fac. Sci., Hokkaido Imp. Univ., Sapporo, Fapan, Ser. II 2, 1-11. (Investigations on snow 10.)

Petrenko, V. F. and R.W.Whitworth. 1999. Physics of ice. Oxford, etc., Oxford University Press.

Priscu, J. C. and 9 others. 1998. Perennial Antarctic lake ice: an oasis for life in a polar desert. Science, 280(5372), 2095-2098.

Schaefer, V.J. and R. J. Cheng. 1968. The effect of the nucleus on ice crystal structure. In International Conference on Cloud Physics, August 26-30, 1968, Toronto, Ont., Canada. Proceedings, 255-259.

Sturm, M. and C. S. Benson. 1997. Vapor transport, grain growth and depth-hoar development in the subarctic snow. F. Glaciol., 43(143), 42-59.

Trabant, D. and C. Benson. 1972. Field experiments on the development of depth hoar. Geol. Soc. Am. Mem. 135, 309-322. 